\title{
AMELIORATIVE EFFECTS OF STEM BARK OF THE WONDER TREE, PROSOPIS CINERARIA (L.) DRUCE AGAINST LPS-INDUCED TOXICITY: AN IN VITRO STUDY
}

\author{
VEENA SHARMA1, PREETI SHARMA² \\ 1,2Department of Bioscience and Biotechnology, Banasthali University, Rajasthan \\ Email: drvsh@gmail.com
}

Received: 10 Aug 2019, Revised and Accepted: 12 Oct 2019

\begin{abstract}
Objective: The present experimental investigation was planned to unravel and analyze the therapeutic potential of hydro-ethanol extract prepared from the stem bark of Prosopis cineraria against LPS-induced toxicity under in vitro conditions.

Methods: Liver tissue samples from healthy Swiss albino male mice (Mus musculus) were used for the study. Liver homogenate ( $0.9 \mathrm{ml})$ was treated with $0.05 \mathrm{mg} / \mathrm{ml}$ of LPS along with 0.01 to $0.05 \mathrm{mg} / \mathrm{ml}$ of hydro-ethanol plant extract and allowed to incubate at $37^{\circ} \mathrm{C}$. The reactions were terminated at different time points at $0 \mathrm{~min}, 30 \mathrm{~min}, 1 \mathrm{~h}, 2 \mathrm{~h}, 4 \mathrm{~h}, 8 \mathrm{~h}$ and $24 \mathrm{~h}$ and alterations in oxidative stress (LPO, CAT, SOD, GSH, GST, and GPx)
\end{abstract} and biochemical parameters of hepatic toxicity (AST and ALT, ACP and ALP) were studied.

Results: The results demonstrated that the obliterations in the levels of oxidative and biochemical parameters due to LPS induced toxicity were restored by the treatment with hydro-ethanol extract of Prosopis cineraria under in vitro conditions. The altered levels were biochemical parameters were observed at $0.05 \mathrm{mg} / \mathrm{ml} \mathrm{LPS}$ concentration after $2 \mathrm{~h}$; but administration of hydro-ethanol plant extract at concentration 0.04 $\mathrm{mg} / \mathrm{ml}$ effectively reduced its level when compared to LPS treated samples under in vitro conditions

Conclusion: The present research work unravelled the alleviating potential of a hydro-ethanol extract of Prosopis cineraria against LPS-induced toxicity by combating oxidative stress under in vitro environment.

Keywords: Antioxidants, In vitro, Prosopis cineraria, Reactive oxygen species, Liver homogenate

(C) 2019 The Authors. Published by Innovare Academic Sciences Pvt Ltd. This is an open-access article under the CC BY license (http://creativecommons.org/licenses/by/4.0/)

DOI: http://dx.doi.org/10.22159/ijcpr.2019v11i6.36352

\section{INTRODUCTION}

Lipopolysaccharides (LPS), also referred to as endotoxins, found in the outer monolayer of most gram-negative bacteria, is known for triggering an innate immune response and inflammatory cascade in the host. The excessive and prolonged inflammatory response triggered by LPS in the host can lead to vascular leakage, septic shock or endotoxin shock, tissue and organ damage and can eventually cause death [1]. LPS has potentially attracted the curiosity of researchers due to its pathogenic role in numerous human and animal diseases [2]. The intensity of the response elicited by LPS depends on its dose and varies from species to species. It is reported that higher animals, humans are more sensitive even at low doses of LPS on the contrary, lower animals are quite resistant to LPS-induced deleterious effects [3]. Studies indicate the massive generation of reactive oxygen species (ROS) in LPS-induced signalling cascade [4]. ROS are known to have biocidal effects on invading micro-organisms and are important components of innate immune response. ROS includes singlet oxygen, superoxide anion radical, highly reactive hydroxyl radical and hydrogen peroxide. However, generation of ROS can potentially harm the tissues and organs of the host $[5,6]$. The lethal effects of LPS are known to be elicited through activation of macrophages, neutrophils which consequently mediate the release of pro-inflammatory cytokines and other bioactive inflammatory mediators, including Tumour Necrosis Factor $\alpha$ (TNF- $\alpha$ ), Interleukin-6 (IL-6) and Nitric oxide (NO). LPS exerts stimulation of antibody production, B-cell proliferation, and activation of T-lymphocytes to produce cytokines [2].

Most common therapeutic treatment for bacterial infections is the administration of antibiotics. But ironically, the use of antibiotics for curing gram-negative bacterial infections can increase endotoxin load and exaggerated inflammatory response. This is due to the fact that antibiotics kill the bacteria but the cell components mainly LPS, continue to elicit an inflammatory response in the host [7]. Thus, in order to develop therapy or effective new drugs for sepsis, the detailed understanding of triggering of inflammatory response and mechanisms underlying the clinical manifestation of serious diseases in the host is indispensable.

India has a tremendous wealth of medicinal plants which are the store-house of various bioactive entities that possess therapeutic properties against numerous diseases. Since ancient times, the importance of plants as a source of medicine has been realized and many medicinal plants are being used as folk medicines for the amelioration of various serious diseases. Prosopis cineraria (L.) Druce, commonly known as 'Khejri', is the state tree of Rajasthan [8]. Prosopis cineraria has therapeutic importance and it is used for curing serious diseases and possess pharmacological activities like anti-fungal, anthelmintic, anti-cancer, anti-bacterial, anti-viral, antihyperglycemic, anti-hyperlipidemic, anti-oxidative [9]. The bark of $P$. cineraria is used in the treatment of various ailments such as asthma, leprosy, wandering of mind, fever, dyspepsia, dysentery, rheumatism, muscle tremors, bronchitis, piles, leucoderma [10, 11]. Numerous Phyto-constituents like tannins, steroids, flavone derivatives (namely Prosogerin A, B, C and E), Rutin, Patulitrin, Luteolin, Patuletin, alkaloids etc. have been reported and isolated from different parts of this medicinal plant [12].

In vitro studies are advantageous as they not only provide indication about the possible in vivo effects of the toxicant or the plant samples under investigation but also reduce time and efforts of the researchers. It must be acknowledged that in vitro studies also help in minimizing the number of animals needed to be sacrificed for the research work. The therapeutic potential of Prosopis cineraria has been extensively explored by many eminent researchers against various serious diseases but its activity against Lipopolysaccharide (endotoxin) is obscure. Therefore, the present research study was executed to elucidate the ameliorative effects of stem bark of Prosopis cineraria against LPS-induced toxicity under in vitro conditions so, that the implication about the possible potential of the plant extract can be deduced with minimal animal sacrifice. 


\section{MATERIALS AND METHODS}

\section{Chemicals}

Lipopolysaccharide (from Escherichia coli 0111:B4) was procured from Sigma-aldrich. The chemicals and all the reagents mentioned in the present experimental research were of high purity (98-99\%) and analytical grade. Chemicals were purchased from reliable firms and sources.

\section{Experimental plant}

The stem-bark of Prosopis cineraria was procured from the local region of Banasthali Vidyapith, Rajasthan. The collected plant was identified by Farm Manager at Krishi Vigyan Kendra, Banasthali Vidyapith, Rajasthan and authenticated at Herbarium unit of Banasthali Vidyapith, Rajasthan, India (Herbarium No.BVRI1359/2017).

\section{Preparation of plant extract}

The plant material i.e. stem bark was shade dried and then powdered by using mixer grinder. The powdered stem-bark (30 g) was packed in a thimble and subjected to soxhlet extraction. The powdered material of the experimental plant was extracted with Pet ether and then the obtained marc was subjected to soxhlet extraction by $80 \%$ ethanol.

The extract was evaporated to dryness using rotary evaporator and stored in airtight jars at $4^{\circ} \mathrm{C}$ for further experimental usage. For experimental use, mixed the plant extracts with the respective solvent to achieve a concentration of $1 \mathrm{mg} / \mathrm{ml}$.

\section{Experimental animals}

The experimental animal model i.e. male Swiss Albino mice (Mus musculus) weighing between 15-30 g were procured from Lala Lajpat Rai University, Hissar (India). (Ref. No. BU/BT/402/14-15).

\section{Maintenance of animal models}

Swiss Albino adult male mice (Mus musculus) were maintained in a duly-ventilated animal house with $12 \mathrm{~h}$ light-dark cycle. Swiss albino mice were housed (six mice per cage) in polypropylene cages in an air-conditioned room with ambient temperature $\left(25 \pm 3^{\circ} \mathrm{C}\right)$, humidity $(50 \pm 15 \%)$. Proper hygienic and sterile conditions were maintained in the animal house facility. The mice were fed with a healthy pelleted diet [Hindustan lever limited, India: metal content in parts per million (ppm) dry weight: $\mathrm{Cu}-10.0, \mathrm{Zn}-45.0, \mathrm{Mn}-55.0$, Co-5.0 and Fe-75.0] and drinking water ad libitum throughout the experimental study.

\section{Liver sample}

Fresh liver samples of healthy Swiss albino male mice weighing approximately $1.6 \mathrm{gm}$ were dissected out after cervical dislocation of the mice.

\section{Preparation of liver homogenate}

$1.6 \mathrm{gm}$ of liver was homogenized in $16 \mathrm{ml}$ of phosphate buffer solution then centrifuged (at $9000 \mathrm{rpm}$ ) for the removal of cell debris for the estimation of various biochemical parameters. During the preparation of liver homogenate temperature was maintained at $4^{\circ} \mathrm{C}$.

\section{LPS solution}

Stock solution of LPS $(1 \mathrm{mg} / \mathrm{ml})$ was prepared by dissolving $1 \mathrm{mg}$ of LPS in $1 \mathrm{ml}$ of double-distilled water.

\section{Experimental regime}

To determine the ameliorative effect of medicinal plant Prosopis cineraria on LPS-induced changes in liver homogenate.

1. Control tubes: Tubes contained $0.9 \mathrm{ml}$ of liver homogenate and $0.1 \mathrm{ml}$ of distilled water.

2. LPS treated tubes: $0.05 \mathrm{ml}$ of LPS was mixed with $0.9 \mathrm{ml}$ of homogenate. The final volume was made upto $1 \mathrm{ml}$ with distilled water.
3. Plant extract treated tubes: $0.05 \mathrm{ml}$ of plant extract was mixed with $0.9 \mathrm{ml}$ of liver homogenate. The final volume was made up to 1 $\mathrm{ml}$ with distilled water.

4. LPS and Plant extract-treated tubes: $0.05 \mathrm{ml}$ of LPS solution and $0.01 \mathrm{ml}$ to $0.05 \mathrm{ml}$ of plant extract were mixed with $0.9 \mathrm{ml}$ of liver homogenate. The final volume was made up to $1 \mathrm{ml}$ with distilled water.

All the tubes were subjected to incubation at $37^{\circ} \mathrm{C}$ and the parameters are analyzed at different time period $-0 \mathrm{~min}, 30 \mathrm{~min}, 1 \mathrm{~h}$, $2 \mathrm{~h}, 4 \mathrm{~h}, 8 \mathrm{~h}$ and $24 \mathrm{~h}$.

\section{Estimation of various oxidative stress and biochemical parameters}

Lipid peroxidation, (LPO) (Nwanjo and Ojiako, 2005) [13]; Superoxide dismutase (SOD) (Marklund and Marklund, 1974) [14];Catalase (CAT) (Aebi,1984) [15]; Reduced glutathione (GSH) (Ellman, 1959) [16]; Glutathione S-transferase (GST) (Habig et al., 1974) [17]; Glutathione peroxidase (GPx) (Rotruck et al., 1973) [18]; Protein estimation (Lowry et al., 1952) [19]; Aspartate transaminase (AST) and Alanine transaminase (Reitman et al., 1957) [20]; Alkaline phosphatase (ALP) and Acid phosphatase (ACP) (Sadashivam, 1996) [21].

\section{Statistical analysis}

The results of the experiments were interpreted as a mean \pm standard deviation of triplicates. The experimental data obtained was analyzed by one way ANOVA (analysis of variance) followed by student's t-test and tukey range test using the SPSS 16.0 (Statistical Program for Social Sciences) program. The experimental results with $\mathrm{p}<0.05$ were considered as satisfactory and $\mathrm{p}<0.001$ were considered as highly significant.

\section{RESULTS}

All the results of the experimental investigation of oxidative stress and biochemical parameters are tabulated in table 1 . Various oxidative stress markers (i.e. LPO, SOD, CAT, GSH, GST and GPx) and biochemical markers (AST and ALT, ALP and ACP) have been studied. Total protein content (TPC) was also estimated to compare the extent of alteration and for the calculation of enzymes specificity with respect to protein content (table 1 (a)). Administration of different concentrations of LPS into liver homogenate of mice led to rise in lipid peroxidation level (LPO) in comparison to control samples (table 1(b)). Our results demonstrated that the addition of $0.05 \mathrm{mg} / \mathrm{ml}$ LPS showed significant alteration in LPO, protein content, SOD, CAT, GSH, GST and GPx. The administration of hydro-ethanol plant extract at concentration $0.04 \mathrm{mg} / \mathrm{ml}$ effectively reduced its level when compared to LPS treated samples under in vitro conditions. Results also showed decreased SOD, CAT and TPC in LPS treated samples in comparison to control tissue samples. SOD, CAT and TPC were positively affected by the treatment of $P$. cineraria. SOD activity was increased in plant treated tissue samples in comparison to LPS intoxicated samples (table 1(c)). Plant extract at $0.04 \mathrm{mg} / \mathrm{ml}$ has capability to restore CAT activity (table $1(\mathrm{~d})$ ). LPS intoxicated liver homogenate samples also showed decrease in GSH content, GST and GPx activities in comparison to control samples of liver tissue. Plant treated liver homogenate samples showed increased GSH content (table 1 (e)), GST activity (table 1 (f)) and GPx (table 1(g)) in comparison to LPS treated liver homogenate. The results highlighted the ameliorative role of hydro-ethanol plant extract of Prosopis cineraria against LPS induced hepatic toxicity by measuring several hepato-toxic markers i.e. AST (Aspartate amino transferase) (table 1 (h)), ALT (Alanine aminotransferase) (table 1 (i)), ACP (Acid Phosphatase) (table (j)) and ALP (Alkaline Phosphatase) (table (k)). The study showed that after $2 \mathrm{~h}$ of incubation the levels of biochemical and oxidative stress parameters were altered noticeably in samples analyzed after $2 \mathrm{~h}$ and $4 \mathrm{~h}$, in comparison to $0 \mathrm{~min}, 30 \mathrm{~min}$ and $1 \mathrm{~h}$ samples. However the samples retrieved after $8 \mathrm{~h}$ and $24 \mathrm{~h}$, the levels of the studied parameters showed marginal difference. The ameliorative effect of bark extract was prominent in the samples retrieved after $2 \mathrm{~h}$ incubation. 
Table 1: Representation of Protein (a), LPO (b) SOD (c), CAT (d), GSH (e), GPx (f), GST (g), AST (h), ALT (i), ACP (j) and ALP (k) levels in liver homogenate treated with different concentrations of plant extract and LPS

(a)

\begin{tabular}{|c|c|c|c|c|c|c|c|c|c|}
\hline \multirow[t]{2}{*}{ Group } & \multirow{2}{*}{$\begin{array}{l}\text { Treatment } \\
\text { LPS(mg/ml) }\end{array}$} & \multirow{2}{*}{$\begin{array}{l}\text { Plant } \\
\text { extract } \\
\text { (mg/ml) }\end{array}$} & \multicolumn{7}{|c|}{ Total protein $(\mathrm{mg} / \mathrm{g})$} \\
\hline & & & at $0 \mathrm{~min}$ & at $30 \mathrm{~min}$ & at $1 \mathrm{~h}$ & at $2 \mathrm{~h}$ & at $4 \mathrm{~h}$ & at $8 \mathrm{~h}$ & at $24 \mathrm{~h}$ \\
\hline Control & - & - & $13.14 \pm 0.07$ & $13.14 \pm 0.08$ & $13.11 \pm 0.79$ & $13.10 \pm 0.10$ & $13.04 \pm 0.71$ & $12.74 \pm 0.12$ & $12.14 \pm 0.73$ \\
\hline LPS Treated & 0.05 & - & $13.13 \pm 0.19$ & $13.03 \pm 0.13$ & $12.43 \pm 0.50$ & $11.64 \pm 0.12$ & $10.03 \pm 0.10^{\mathrm{a}}$ & $09.63 \pm 0.14$ & $09.43 \pm 0.19^{*}$ \\
\hline Plant Treated & - & 0.05 & $13.12 \pm 0.20$ & $13.01 \pm 0.10^{*}$ & $13.23 \pm 0.12$ & $13.04 \pm 0.43$ & $13.06 \pm 0.16$ & $12.93 \pm 0.18$ & $12.83 \pm 0.29$ \\
\hline LPS+Plant & 0.05 & 0.01 & $13.13 \pm 0.11$ & $13.06 \pm 0.47$ & $12.13 \pm 0.53$ & $11.09 \pm 0.14^{*}$ & $10.73 \pm 0.12$ & $09.65 \pm 0.13$ & $09.13 \pm 0.21$ \\
\hline \multirow{4}{*}{ treated } & & 0.02 & $13.13 \pm 0.29$ & $13.03 \pm 0.18$ & $12.91 \pm 0.64$ & $11.68 \pm 0.11^{*}$ & $10.93 \pm 0.17$ & $10.63 \pm 0.27$ & $09.93 \pm 0.26$ \\
\hline & & 0.03 & $13.13 \pm 0.22$ & $13.02 \pm 0.10$ & $12.43 \pm 0.55$ & $11.64 \pm 0.10$ & $10.53 \pm 0.19^{\mathrm{a}}$ & $09.61 \pm 0.49$ & $09.54 \pm 0.21$ \\
\hline & & 0.04 & $13.13 \pm 0.14$ & $13.01 \pm 0.29$ & $12.92 \pm 0.36$ & $11.94 \pm 0.13$ & $11.13 \pm 0.24$ & $11.02 \pm 0.79^{a}$ & $10.43 \pm 0.24$ \\
\hline & & 0.05 & $13.13 \pm 0.25$ & $13.03 \pm 0.21$ & $12.13 \pm 0.51^{*}$ & $11.60 \pm 0.18$ & $10.43 \pm 0.21$ & $10.13 \pm 0.23$ & $10.07 \pm 0.29 *$ \\
\hline
\end{tabular}

Values are represented as mean $\pm \mathrm{SD}(\mathrm{n}=6) .{ }^{*} \mathrm{p}<0.05,{ }^{* *} \mathrm{p}<0.001 \mathrm{v} / \mathrm{s}$ control group; ${ }^{\mathrm{a}} \mathrm{p}<0.05,{ }^{\mathrm{b}} \mathrm{p}<0.001 \mathrm{v} / \mathrm{s}$ LPS treated group.

(b)

\begin{tabular}{|c|c|c|c|c|c|c|c|c|c|}
\hline \multirow[t]{2}{*}{ Group } & \multirow{2}{*}{$\begin{array}{l}\text { Treatment } \\
\text { LPS(mg/ml) }\end{array}$} & \multirow{2}{*}{$\begin{array}{l}\text { Plant extract } \\
(\mathrm{mg} / \mathrm{ml})\end{array}$} & \multicolumn{7}{|c|}{ LPO (nmolesof MDA formed/mg tissue) } \\
\hline & & & at $0 \mathrm{~min}$ & at $30 \mathrm{~min}$ & at $1 \mathrm{~h}$ & at $2 \mathrm{~h}$ & at $4 \mathrm{~h}$ & at $8 \mathrm{~h}$ & at $24 \mathrm{~h}$ \\
\hline Control & - & - & $12.14 \pm 0.77$ & $12.16 \pm 0.08$ & $12.22 \pm 0.12$ & $12.24 \pm 0.10$ & $12.24 \pm 0.71$ & $12.19 \pm 0.12$ & $12.17 \pm 0.13$ \\
\hline LPS Treated & 0.05 & - & $12.16 \pm 0.13$ & $12.12 \pm 0.24$ & $18.75 \pm 0.26^{*}$ & $21.52 \pm 0.16$ & $23.12 \pm 0.37$ & $23.70 \pm 0.88^{\mathrm{a}}$ & $23.81 \pm 0.19$ \\
\hline Plant Treated & - & 0.05 & $12.19 \pm 0.14$ & $12.32 \pm 0.35$ & $12.72 \pm 0.17$ & $12.50 \pm 0.10$ & $13.22 \pm 0.18$ & $13.71 \pm 0.11$ & $13.12 \pm 0.20^{*}$ \\
\hline LPS+Plant & 0.05 & 0.01 & $12.15 \pm 0.15$ & $12.92 \pm 0.14$ & $18.72 \pm 0.18$ & $21.22 \pm 0.11$ & $23.42 \pm 0.19 *$ & $23.12 \pm 0.09$ & $23.82 \pm 0.24$ \\
\hline \multirow[t]{4}{*}{ treated } & & 0.02 & $12.12 \pm 0.16$ & $12.92 \pm 0.27$ & $18.72 \pm 0.49$ & $21.52 \pm 0.19$ & $23.52 \pm 0.10$ & $23.72 \pm 0.21^{*}$ & $23.82 \pm 0.02$ \\
\hline & & 0.03 & $12.15 \pm 0.37$ & $12.92 \pm 0.18$ & $18.72 \pm 0.20$ & $21.52 \pm 0.25$ & $23.52 \pm 0.21^{\mathrm{a}}$ & $23.72 \pm 0.27$ & $23.82 \pm 0.23$ \\
\hline & & 0.04 & $12.19 \pm 0.18$ & $12.95 \pm 0.11$ & $19.12 \pm 0.20$ & $19.10 \pm 0.01 *$ & $17.45 \pm 0.22$ & $17.19 \pm 0.13$ & $16.09 \pm 0.24^{*}$ \\
\hline & & 0.05 & $12.14 \pm 0.11$ & $12.65 \pm 0.21$ & $18.15 \pm 0.23$ & $17.35 \pm 0.12$ & $16.18 \pm 0.20$ & $16.11 \pm 0.14^{*}$ & $15.15 \pm 0.25$ \\
\hline
\end{tabular}

Values are represented as mean $\pm \mathrm{SD}(\mathrm{n}=6) .{ }^{*} \mathrm{p}<0.05,{ }^{* *} \mathrm{p}<0.001 \mathrm{v} / \mathrm{s}$ control group; ${ }^{\mathrm{p}}<0.05,{ }^{\mathrm{b}} \mathrm{p}<0.001 \mathrm{v} / \mathrm{s}$ LPS treated group. (LPO-Lipid peroxidation)

(c)

\begin{tabular}{|c|c|c|c|c|c|c|c|c|c|}
\hline \multirow[t]{2}{*}{ Group } & \multirow{2}{*}{$\begin{array}{l}\text { Treatment } \\
\text { LPS }(\mathrm{mg} / \mathrm{ml})\end{array}$} & \multirow{2}{*}{$\begin{array}{l}\text { Plant } \\
\text { extract } \\
(\mathrm{mg} / \mathrm{ml})\end{array}$} & \multicolumn{7}{|c|}{$\operatorname{SOD}(\mathrm{U} / \mathrm{min} / \mathrm{mg}$ protein $)$} \\
\hline & & & at $0 \mathrm{~min}$ & $\begin{array}{l}\text { at } 30 \\
\text { min }\end{array}$ & at $1 \mathrm{~h}$ & at $2 \mathrm{~h}$ & at $4 \mathrm{~h}$ & at $8 \mathrm{~h}$ & at $24 \mathrm{~h}$ \\
\hline Control & - & - & $0.05 \pm 0.70$ & $0.05 \pm 0.08$ & $0.04 \pm 0.78$ & $0.04 \pm 0.12$ & $0.04 \pm 0.18$ & $0.04 \pm 0.05$ & $0.04 \pm 0.98$ \\
\hline LPS Treated & 0.05 & - & $0.05 \pm 0.73$ & $0.04 \pm 0.11$ & $0.03 \pm 0.13$ & $0.03 \pm 0.16^{*}$ & $0.02 \pm 0.11$ & $0.02 \pm 0.16$ & $0.02 \pm 0.13^{*}$ \\
\hline Plant Treated & - & 0.05 & $0.05 \pm 0.76$ & $0.05 \pm 0.08$ & $0.04 \pm 0.78$ & $0.04 \pm 0.12$ & $0.04 \pm 0.18$ & $0.03 \pm 0.05^{*}$ & $0.03 \pm 0.98$ \\
\hline LPS+Plant treated & 0.05 & 0.01 & $0.05 \pm 0.79$ & $0.04 \pm 0.12$ & $0.03 \pm 0.13$ & $0.03 \pm 0.16$ & $0.02 \pm 0.11^{*}$ & $0.02 \pm 0.16$ & $0.02 \pm 0.23$ \\
\hline & & 0.02 & $0.05 \pm 0.82$ & $0.04 \pm 0.08$ & $0.04 \pm 0.78$ & $0.03 \pm 0.12$ & $0.03 \pm 0.18$ & $0.02 \pm 0.05$ & $0.02 \pm 0.98 *$ \\
\hline & & 0.03 & $0.05 \pm 0.85$ & $0.04 \pm 0.13$ & $0.03 \pm 0.13$ & $0.03 \pm 0.16$ & $0.02 \pm 0.11^{\mathrm{a}}$ & $0.02 \pm 0.16$ & $0.02 \pm 0.13$ \\
\hline & & 0.04 & $0.05 \pm 0.88$ & $0.05 \pm 0.08$ & $0.05 \pm 0.78$ & $0.04 \pm 0.12^{*}$ & $0.04 \pm 0.18$ & $0.04 \pm 0.05$ & $0.03 \pm 0.98^{*}$ \\
\hline & & 0.05 & $0.05 \pm 0.91$ & $0.05 \pm 0.14$ & $0.05 \pm 0.13$ & $0.04 \pm 0.16^{*}$ & $0.04 \pm 0.11$ & $0.04 \pm 0.16$ & $0.04 \pm 0.13$ \\
\hline
\end{tabular}

Values are represented as mean $\pm \mathrm{SD}(\mathrm{n}=6) .{ }^{*} \mathrm{p}<0.05,{ }^{*} \mathrm{p}<0.001 \mathrm{v} / \mathrm{s}$ control group; ${ }^{\mathrm{a}} \mathrm{p}<0.05,{ }^{\mathrm{b}} \mathrm{p}<0.001 \mathrm{v} / \mathrm{s}$ LPS treated group. (SOD-Superoxide dismutase)

(d)

\begin{tabular}{|c|c|c|c|c|c|c|c|c|c|}
\hline \multirow[t]{2}{*}{ Group } & \multirow{2}{*}{$\begin{array}{l}\text { Treatment } \\
\text { LPS }(\mathrm{mg} / \mathrm{ml})\end{array}$} & \multirow{2}{*}{$\begin{array}{l}\text { Plant } \\
\text { extract } \\
\text { (mg/ml) }\end{array}$} & \multicolumn{7}{|c|}{ CAT( $\mu$ mole of $\mathrm{H} 202$ consumed $/ \mathrm{min} / \mathrm{mg}$ protein) } \\
\hline & & & at $0 \mathrm{~min}$ & at $30 \mathrm{~min}$ & at $1 \mathrm{~h}$ & at $2 \mathrm{~h}$ & at $4 \mathrm{~h}$ & at $8 \mathrm{~h}$ & at $24 \mathrm{~h}$ \\
\hline Control & - & - & $155.94 \pm 0.74$ & $155.84 \pm 0.15$ & $153.12 \pm 0.86$ & $152.14 \pm 0.71$ & $151.14 \pm 0.32$ & $151.03 \pm 0.19$ & $150.14 \pm 0.80$ \\
\hline LPS Treated & 0.05 & - & $155.14 \pm 0.17$ & $151.98 \pm 0.36$ & $142.20 \pm 0.65$ & $139.72 \pm 0.16^{*}$ & $136.72 \pm 0.77^{*}$ & $134.72 \pm 0.38$ & $134.12 \pm 0.10$ \\
\hline Plant Treated & & 0.05 & $154.19 \pm 0.40$ & $154.11 \pm 0.10$ & $152.34 \pm 0.16$ & $152.04 \pm 0.11$ & $152.24 \pm 0.39$ & $151.11 \pm 0.11$ & $151.04 \pm 0.86$ \\
\hline LPS+Plant treated & 0.05 & 0.01 & $152.10 \pm 0.97$ & $151.91 \pm 0.37$ & $142.21 \pm 0.66$ & $139.82 \pm 0.17 *$ & $136.12 \pm 0.78$ & $134.92 \pm 0.39$ & $134.02 \pm 0.11$ \\
\hline & & 0.02 & $152.11 \pm 0.15$ & $152.04 \pm 0.15$ & $149.19 \pm 0.86$ & $139.04 \pm 0.71$ & $138.18 \pm 0.32$ & $137.14 \pm 0.19$ & $132.04 \pm 0.80^{*}$ \\
\hline & & 0.03 & $152.14 \pm 0.21$ & $151.98 \pm 0.38$ & $142.20 \pm 0.67$ & $139.72 \pm 0.18$ & $136.72 \pm 0.79$ & $134.72 \pm 0.40$ & $134.12 \pm 0.12$ \\
\hline & & 0.04 & $155.94 \pm 0.26$ & $151.10 \pm 0.15$ & $148.14 \pm 0.46$ & $152.19 \pm 0.01 *$ & $153.14 \pm 0.32^{\mathrm{a}}$ & $152.14 \pm 0.19$ & $152.84 \pm 0.80$ \\
\hline & & 0.05 & $155.74 \pm 0.32$ & $151.98 \pm 0.39$ & $142.20 \pm 0.68$ & $150.72 \pm 0.19 \mathrm{a}$ & $151.72 \pm 0.80^{*}$ & $154.72 \pm 0.41$ & $154.12 \pm 0.13$ \\
\hline
\end{tabular}

Values are represented as mean $\pm \mathrm{SD}(\mathrm{n}=6) .{ }^{*} \mathrm{p}<0.05,{ }^{* *} \mathrm{p}<0.001 \mathrm{v} / \mathrm{s}$ control group; ${ }^{\mathrm{a}} \mathrm{p}<0.05,{ }^{\mathrm{b}} \mathrm{p}<0.001 \mathrm{v} / \mathrm{s}$ LPS treated group. (CAT-Catalase)

(e)

\begin{tabular}{|c|c|c|c|c|c|c|c|c|c|}
\hline \multirow[t]{2}{*}{ Group } & \multirow{2}{*}{$\begin{array}{l}\text { Treatment } \\
\text { LPS(mg/ml) }\end{array}$} & \multirow{2}{*}{$\begin{array}{l}\text { Plant } \\
\text { extract } \\
\text { (mg/ml) }\end{array}$} & \multicolumn{7}{|c|}{ GSH $(\mu$ mole GSH $/ g$ tissue $)$} \\
\hline & & & at $0 \mathrm{~min}$ & $\begin{array}{l}\text { at } 30 \\
\text { min }\end{array}$ & at $1 \mathrm{~h}$ & at $2 \mathrm{~h}$ & at $4 \mathrm{~h}$ & at $8 \mathrm{~h}$ & at $24 \mathrm{~h}$ \\
\hline Control & - & - & $3.16 \pm 0.12$ & $3.16 \pm 0.93$ & $3.15 \pm 0.74$ & $3.14 \pm 0.25$ & $3.14 \pm 0.96$ & $3.13 \pm 0.37$ & $3.13 \pm 0.78$ \\
\hline LPS Treated & 0.05 & - & $3.15 \pm 0.75$ & $3.08 \pm 0.44$ & $3.03 \pm 0.95$ & $3.00 \pm 0.06 *$ & $2.98 \pm 0.17$ & $2.96 \pm 0.18$ & $2.95 \pm 0.19$ \\
\hline Plant Treated & - & 0.05 & $3.15 \pm 0.138$ & $3.15 \pm 0.93$ & $3.14 \pm 0.74$ & $3.14 \pm 0.25$ & $3.14 \pm 0.96$ & $3.14 \pm 0.37$ & $3.13 \pm 0.78$ \\
\hline \multirow{5}{*}{ LPS+Plant treated } & 0.05 & 0.01 & $3.15 \pm 0.01$ & $3.09 \pm 0.45$ & $3.02 \pm 0.96$ & $3.02 \pm 0.07$ & $2.99 \pm 0.18^{*}$ & $2.92 \pm 0.19$ & $2.91 \pm 0.20$ \\
\hline & & 0.02 & $3.17 \pm 0.24$ & $3.15 \pm 0.91$ & $3.04 \pm 0.71$ & $3.04 \pm 0.15$ & $2.94 \pm 0.16$ & $3.04 \pm 0.31$ & $3.01 \pm 0.82$ \\
\hline & & 0.03 & $3.15 \pm 0.37$ & $3.08 \pm 0.46$ & $3.03 \pm 0.97$ & $3.00 \pm 0.08$ & $2.98 \pm 0.19 *$ & $2.96 \pm 0.20$ & $2.95 \pm 0.21$ \\
\hline & & 0.04 & $3.18 \pm 0.390$ & $3.15 \pm 0.93$ & $3.15 \pm 0.74$ & $3.14 \pm 0.25^{*}$ & $3.14 \pm 0.96$ & $3.14 \pm 0.37$ & $3.14 \pm 0.78$ \\
\hline & & 0.05 & $3.16 \pm 0.453$ & $3.08 \pm 0.47$ & $3.03 \pm 0.98$ & $3.00 \pm 0.09$ & $3.08 \pm 0.20$ & $3.06 \pm 0.21^{*}$ & $3.15 \pm 0.22$ \\
\hline
\end{tabular}

Values are represented as mean $\pm \mathrm{SD}(\mathrm{n}=6) .{ }^{*} \mathrm{p}<0.05,{ }^{* *} \mathrm{p}<0.001 \mathrm{v} / \mathrm{s}$ control group; ${ }^{\mathrm{p}} \mathrm{p}<0.05,{ }^{\mathrm{b}} \mathrm{p}<0.001 \mathrm{v} / \mathrm{s}$ LPS treated group. (GSH-Reduced glutathione) 
(f)

\begin{tabular}{|c|c|c|c|c|c|c|c|c|c|}
\hline \multirow[t]{2}{*}{ Group } & \multirow{2}{*}{$\begin{array}{l}\text { Treatment } \\
\text { LPS }(\mathrm{mg} / \mathrm{ml})\end{array}$} & \multirow{2}{*}{$\begin{array}{l}\text { Plant } \\
\text { extract } \\
(\mathrm{mg} / \mathrm{ml})\end{array}$} & \multicolumn{7}{|c|}{ GST(nmole CDNB-GSH conjugate formed $/ \mathrm{min} / \mathrm{mg}$ protein) } \\
\hline & & & at $0 \mathrm{~min}$ & at $30 \mathrm{~min}$ & at $1 \mathrm{~h}$ & at $2 \mathrm{~h}$ & at $4 \mathrm{~h}$ & at $8 \mathrm{~h}$ & at $24 \mathrm{~h}$ \\
\hline Control & - & - & $525.04 \pm 0.74$ & $525.54 \pm 0.15$ & $523.01 \pm 0.70$ & $523.11 \pm 0.17$ & $521.29 \pm 0.11$ & $520.11 \pm 0.79$ & $520.04 \pm 0.10$ \\
\hline LPS Treated & 0.05 & - & $524.94 \pm 0.77$ & $511.17 \pm 0.08$ & $500.14 \pm 0.19$ & $495.14 \pm 0.10^{*}$ & $493.14 \pm 0.81$ & $490.14 \pm 0.02$ & $485.14 \pm 0.13$ \\
\hline Plant Treated & - & 0.05 & $526.14 \pm 0.80$ & $525.85 \pm 0.75$ & $525.13 \pm 0.26$ & $524.24 \pm 0.47$ & $524.94 \pm 0.78$ & $523.19 \pm 0.29$ & $523.23 \pm 0.80$ \\
\hline \multirow[t]{5}{*}{ LPS+Plant treated } & 0.05 & 0.01 & $525.10 \pm 0.83$ & $521.14 \pm 0.79$ & $520.53 \pm 0.30$ & $497.14 \pm 0.40$ & $495.14 \pm 0.81$ & $496.11 \pm 0.32^{*}$ & $498.89 \pm 0.83$ \\
\hline & & 0.02 & $524.21 \pm 0.86$ & $520.13 \pm 0.75$ & $520.17 \pm 0.39 *$ & $520.34 \pm 0.73$ & $520.04 \pm 0.38$ & $520.04 \pm 0.19$ & $520.45 \pm 0.34$ \\
\hline & & 0.03 & $525.12 \pm 0.89$ & $515.12 \pm 0.10$ & $517.18 \pm 0.71$ & $515.11 \pm 0.07^{*}$ & $518.24 \pm 0.81$ & $521.14 \pm 0.12$ & $525.31 \pm 0.83$ \\
\hline & & 0.04 & $526.15 \pm 0.92$ & $525.14 \pm 0.15$ & $525.16 \pm 0.76$ & $524.14 \pm 0.43^{*}$ & $523.14 \pm 0.78$ & $523.04 \pm 0.19^{a}$ & $520.13 \pm 0.80$ \\
\hline & & 0.05 & $524.17 \pm 0.95$ & $521.10 \pm 0.81$ & $520.12 \pm 0.84$ & $525.19 \pm 0.60 *$ & $525.54 \pm 0.21$ & $529.21 \pm 0.12$ & $531.04 \pm 0.83^{*}$ \\
\hline
\end{tabular}

Values are represented as mean $\pm \mathrm{SD}(\mathrm{n}=6) .{ }^{*} \mathrm{p}<0.05,{ }^{* *} \mathrm{p}<0.001 \mathrm{v} / \mathrm{s}$ control group; ${ }^{\mathrm{a}} \mathrm{p}<0.05,{ }^{\mathrm{b}} \mathrm{p}<0.001 \mathrm{v} / \mathrm{s}$ LPS treated group. (GST-Glutathione-stransferase)

(g)

\begin{tabular}{|c|c|c|c|c|c|c|c|c|c|}
\hline \multirow[t]{2}{*}{ Group } & \multirow{2}{*}{$\begin{array}{l}\text { Treatment } \\
\text { LPS(mg/ml) }\end{array}$} & \multirow{2}{*}{$\begin{array}{l}\text { Plant } \\
\text { extract } \\
(\mathrm{mg} / \mathrm{ml})\end{array}$} & \multicolumn{7}{|c|}{ GPx(nM NADH oxidised/mg protein) } \\
\hline & & & at $0 \mathrm{~min}$ & at $30 \mathrm{~min}$ & at $1 \mathrm{~h}$ & at $2 \mathrm{~h}$ & at $4 \mathrm{~h}$ & at $8 \mathrm{~h}$ & at $24 \mathrm{~h}$ \\
\hline Control & - & - & $26.24 \pm 0.37$ & $26.04 \pm 0.08$ & $26.10 \pm 0.39$ & $25.09 \pm 0.40$ & $25.06 \pm 0.51$ & $25.14 \pm 0.92$ & $24.04 \pm 0.13$ \\
\hline LPS Treated & 0.05 & - & $25.10 \pm 0.40$ & $23.64 \pm 0.48$ & $20.44 \pm 0.34$ & $19.17 \pm 0.70$ & $19.19 \pm 0.47$ & $18.14 \pm 0.12$ & $17.91 \pm 0.03^{*}$ \\
\hline Plant Treated & - & 0.05 & $25.24 \pm 0.38$ & $25.14 \pm 0.09$ & $25.10 \pm 0.40$ & $24.09 \pm 0.11$ & $24.06 \pm 0.52$ & $24.14 \pm 0.93$ & $24.94 \pm 0.14$ \\
\hline \multirow[t]{5}{*}{ LPS+Plant treated } & 0.05 & 0.01 & $25.17 \pm 0.41$ & $23.11 \pm 0.49$ & $20.14 \pm 0.31$ & $19.18 \pm 0.70$ & $19.34 \pm 0.41$ & $19.04 \pm 0.12^{a}$ & $19.19 \pm 0.93$ \\
\hline & & 0.02 & $24.14 \pm 0.39$ & $24.14 \pm 0.10$ & $23.10 \pm 0.41$ & $22.09 \pm 0.12^{*}$ & $21.06 \pm 0.53$ & $20.14 \pm 0.94^{\mathrm{a}}$ & $20.94 \pm 0.15$ \\
\hline & & 0.03 & $25.10 \pm 0.82$ & $24.94 \pm 0.50$ & $24.45 \pm 0.32$ & $23.11 \pm 0.43$ & $22.14 \pm 0.90^{*}$ & $20.18 \pm 0.12$ & $19.34 \pm 0.43$ \\
\hline & & 0.04 & $25.24 \pm 0.40$ & $24.04 \pm 0.61$ & $24.04 \pm 0.45$ & $24.21 \pm 0.13^{*}$ & $23.29 \pm 0.44$ & $22.19 \pm 0.40$ & $20.18 \pm 0.16$ \\
\hline & & 0.05 & $25.04 \pm 0.38$ & $24.11 \pm 0.09$ & $24.10 \pm 0.40^{\mathrm{a}}$ & $24.09 \pm 0.11$ & $23.06 \pm 0.52$ & $23.14 \pm 0.93$ & $20.94 \pm 0.14$ \\
\hline
\end{tabular}

Values are represented as mean $\pm \mathrm{SD}(\mathrm{n}=6) .{ }^{*} \mathrm{p}<0.05,{ }^{*} \mathrm{p}<0.001 \mathrm{v} / \mathrm{s}$ control group; ${ }^{\mathrm{a}} \mathrm{p}<0.05,{ }^{\mathrm{b}} \mathrm{p}<0.001 \mathrm{v} / \mathrm{s}$ LPS treated group. (GPx-Glutathione peroxidase)

(h)

\begin{tabular}{|c|c|c|c|c|c|c|c|c|c|}
\hline \multirow[t]{2}{*}{ Group } & \multirow{2}{*}{$\begin{array}{l}\text { Treatment } \\
\text { LPS }(\mathrm{mg} / \mathrm{ml})\end{array}$} & \multirow{2}{*}{$\begin{array}{l}\text { Plant } \\
\text { extract } \\
(\mathrm{mg} / \mathrm{ml})\end{array}$} & \multicolumn{7}{|c|}{ 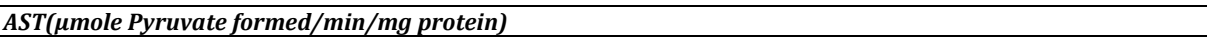 } \\
\hline & & & at $0 \mathrm{~min}$ & at $30 \mathrm{~min}$ & at $1 \mathrm{~h}$ & at $2 \mathrm{~h}$ & at $4 \mathrm{~h}$ & at $8 \mathrm{~h}$ & at $24 \mathrm{~h}$ \\
\hline Control & - & - & $195.55 \pm 0.77$ & $192.14 \pm 0.08$ & $192.90 \pm 0.19$ & $190.24 \pm 0.40$ & $188.64 \pm 0.81$ & $186.51 \pm 0.22$ & $182.03 \pm 0.13$ \\
\hline LPS Treated & 0.05 & - & $196.50 \pm 0.80$ & $200.28 \pm 0.44$ & $219.30 \pm 0.40$ & $224.07 \pm 0.18^{*}$ & $228.51 \pm 0.39$ & $233.54 \pm 0.23$ & $237.17 \pm 0.11$ \\
\hline Plant Treated & - & 0.05 & $198.14 \pm 0.63$ & $196.07 \pm 0.08$ & $196.21 \pm 0.71$ & $199.54 \pm 0.89$ & $198.59 \pm 0.01$ & $192.14 \pm 0.22$ & $192.04 \pm 0.13$ \\
\hline \multirow{5}{*}{ LPS+Plant treated } & 0.05 & 0.01 & $197.24 \pm 0.16$ & $198.25 \pm 0.45$ & $217.38 \pm 0.41$ & $220.07 \pm 0.18$ & $221.51 \pm 0.16$ & $230.56 \pm 0.20$ & $232.37 \pm 0.29 *$ \\
\hline & & 0.02 & $197.50 \pm 0.09$ & $192.14 \pm 0.78$ & $212.59 \pm 0.09$ & $210.04 \pm 0.89^{a}$ & $208.24 \pm 0.81 *$ & $223.01 \pm 0.12$ & $220.59 \pm 0.03$ \\
\hline & & 0.03 & $195.59 \pm 0.02$ & $194.28 \pm 0.46$ & $210.30 \pm 0.42 *$ & $200.57 \pm 0.18$ & $198.07 \pm 0.19$ & $196.17 \pm 0.20$ & $195.52 \pm 0.21$ \\
\hline & & 0.04 & $195.14 \pm 0.15$ & $194.54 \pm 0.06$ & $201.94 \pm 0.97$ & $192.53 \pm 0.78$ & $191.04 \pm 0.09$ & $188.84 \pm 0.17$ & $182.02 \pm 0.10$ \\
\hline & & 0.05 & $196.74 \pm 0.28$ & $198.28 \pm 0.47$ & $195.30 \pm 0.43$ & $199.59 \pm 0.18$ & $200.53 \pm 0.09$ & $187.97 \pm 0.20$ & $181.07 \pm 0.01$ \\
\hline
\end{tabular}

Values are represented as mean $\pm \mathrm{SD}(\mathrm{n}=6) .{ }^{*} \mathrm{p}<0.05,{ }^{* *} \mathrm{p}<0.001 \mathrm{v} / \mathrm{s}$ control group; ${ }^{\mathrm{p}} \mathrm{p}<0.05,{ }^{\mathrm{b}} \mathrm{p}<0.001 \mathrm{v} / \mathrm{s}$ LPS treated group. (AST-aspartate transferase).

(i)

\begin{tabular}{|c|c|c|c|c|c|c|c|c|c|}
\hline \multirow[t]{2}{*}{ Group } & \multirow{2}{*}{$\begin{array}{l}\text { Treatment } \\
\text { LPS } \\
(\mathrm{mg} / \mathrm{ml})\end{array}$} & \multirow{2}{*}{$\begin{array}{l}\text { Plant } \\
\text { extract } \\
\text { (mg/ml) }\end{array}$} & \multicolumn{7}{|c|}{ ALT( $\mu$ mole Pyruvate formed $/ \mathrm{min} / \mathrm{mg}$ protein) } \\
\hline & & & at $0 \mathrm{~min}$ & at $30 \mathrm{~min}$ & at $1 \mathrm{~h}$ & at $2 \mathrm{~h}$ & at $4 \mathrm{~h}$ & at $8 \mathrm{~h}$ & at $24 \mathrm{~h}$ \\
\hline Control & - & - & $343.40 \pm 0.77$ & $354.14 \pm 0.08$ & $361.04 \pm 0.72$ & $369.14 \pm 0.80$ & $367.46 \pm 0.11$ & $364.04 \pm 0.02$ & $361.67 \pm 0.89$ \\
\hline LPS Treated & 0.05 & - & $347.94 \pm 0.80$ & $350.57 \pm 0.31$ & $377.30 \pm 0.40$ & $380.57 \pm 0.18$ & $386.58 \pm 0.11$ & $388.07 \pm 0.20^{a}$ & $390.51 \pm 0.11$ \\
\hline Plant Treated & - & 0.05 & $347.18 \pm 0.83$ & $357.14 \pm 0.68$ & $363.14 \pm 0.39$ & $367.12 \pm 0.80$ & $365.10 \pm 0.11$ & $367.84 \pm 0.82$ & $360.10 \pm 0.53$ \\
\hline \multirow[t]{5}{*}{ LPS+Plant treated } & 0.05 & 0.01 & $350.21 \pm 0.06$ & $352.50 \pm 0.32$ & $372.30 \pm 0.41$ & $377.87 \pm 0.08$ & $380.57 \pm 0.19$ & $381.97 \pm 0.90$ & $382.56 \pm 0.21$ \\
\hline & & 0.02 & $347.17 \pm 0.89$ & $357.94 \pm 0.78$ & $371.04 \pm 0.09$ & $377.19 \pm 0.80$ & $377.14 \pm 0.89$ & $371.84 \pm 0.12$ & $377.15 \pm 0.93$ \\
\hline & & 0.03 & $348.14 \pm 0.02$ & $360.58 \pm 0.33$ & $377.30 \pm 0.42$ & $380.67 \pm 0.18^{*}$ & $380.12 \pm 0.89$ & $383.57 \pm 0.20$ & $387.57 \pm 0.01$ \\
\hline & & 0.04 & $348.11 \pm 0.95$ & $367.04 \pm 0.06$ & $371.84 \pm 0.17 *$ & $377.14 \pm 0.08$ & $369.18 \pm 0.19$ & $360.94 \pm 0.10$ & $367.19 \pm 0.11$ \\
\hline & & 0.05 & $347.17 \pm 0.08$ & $367.14 \pm 0.99$ & $371.04 \pm 0.10$ & $375.18 \pm 0.01$ & $361.14 \pm 0.72$ & $369.34 \pm 0.17$ & $366.94 \pm 0.14$ \\
\hline
\end{tabular}

Values are represented as mean $\pm \mathrm{SD}(\mathrm{n}=6){ }^{*} \mathrm{p}<0.05,{ }^{* *} \mathrm{p}<0.001 \mathrm{v} / \mathrm{s}$ control group; ${ }^{\mathrm{a}} \mathrm{p}<0.05,{ }^{\mathrm{b}} \mathrm{p}<0.001 \mathrm{v} / \mathrm{s}$ LPS treated group. (ALT-alanine transferase).

(j)

\begin{tabular}{|c|c|c|c|c|c|c|c|c|c|}
\hline \multirow[t]{2}{*}{ Group } & \multirow{2}{*}{$\begin{array}{l}\text { Treatment } \\
\text { LPS(mg/ml) }\end{array}$} & \multirow{2}{*}{$\begin{array}{l}\text { Plant } \\
\text { extract } \\
(\mathrm{mg} / \mathrm{ml})\end{array}$} & \multicolumn{7}{|c|}{ ACP $(\mu \mathrm{mole} p \mathrm{pP}$ released $/ \mathrm{min} / \mathrm{mg}$ protein $)$} \\
\hline & & & at $0 \mathrm{~min}$ & at $30 \mathrm{~min}$ & at $1 \mathrm{~h}$ & at $2 \mathrm{~h}$ & at $4 \mathrm{~h}$ & at $8 \mathrm{~h}$ & at $24 \mathrm{~h}$ \\
\hline Control & - & - & $5.14 \pm 0.17$ & $5.12 \pm 0.11$ & $5.06 \pm 0.19$ & $4.90 \pm 0.23$ & $4.99 \pm 0.21$ & $4.95 \pm 0.22$ & $4.90 \pm 0.13$ \\
\hline LPS Treated & 0.05 & - & $4.94 \pm 0.20$ & $4.09 \pm 0.14$ & $4.01 \pm 0.95^{*}$ & $3.97 \pm 0.04$ & $3.82 \pm 0.62$ & $3.77 \pm 0.18$ & $3.67 \pm 0.19$ \\
\hline Plant Treated & - & 0.05 & $4.94 \pm 0.11$ & $4.09 \pm 0.15$ & $4.01 \pm 0.16$ & $4.74 \pm 0.66$ & $4.70 \pm 0.67$ & $4.50 \pm 0.68$ & $4.17 \pm 0.60$ \\
\hline \multirow{5}{*}{ LPS+Plant treated } & 0.05 & 0.01 & $4.19 \pm 0.22$ & $4.19 \pm 0.36$ & $4.11 \pm 0.97$ & $3.99 \pm 0.16$ & $3.96 \pm 0.58$ & $3.97 \pm 0.62$ & $3.95 \pm 0.19$ \\
\hline & & 0.02 & $4.17 \pm 0.23$ & $4.39 \pm 0.17$ & $4.41 \pm 0.18$ & $4.07 \pm 0.66^{*}$ & $4.06 \pm 0.67$ & $4.17 \pm 0.68$ & $3.94 \pm 0.19$ \\
\hline & & 0.03 & $4.94 \pm 0.04$ & $4.45 \pm 0.18$ & $4.54 \pm 0.99$ & $4.16 \pm 0.09 *$ & $4.01 \pm 0.14$ & $3.99 \pm 0.08^{\mathrm{a}}$ & $3.97 \pm 0.63$ \\
\hline & & 0.04 & $4.55 \pm 0.15$ & $4.61 \pm 0.26^{\mathrm{a}}$ & $4.74 \pm 0.17$ & $4.82 \pm 0.28^{*}$ & $4.14 \pm 0.09$ & $4.10 \pm 0.30$ & $4.11 \pm 0.11$ \\
\hline & & 0.05 & $4.84 \pm 0.20$ & $4.74 \pm 0.21$ & $4.72 \pm 0.29$ & $4.80 \pm 0.85$ & $4.24 \pm 0.83$ & $4.17 \pm 0.30^{\mathrm{a}}$ & $4.19 \pm 0.39$ \\
\hline
\end{tabular}

Values are represented as mean $\pm \mathrm{SD}(\mathrm{n}=6){ }^{*} \mathrm{p}<0.05,{ }^{* *} \mathrm{p}<0.001 \mathrm{v} / \mathrm{s}$ control group; a $<<0.05,{ }^{b} \mathrm{p}<0.001 \mathrm{v} / \mathrm{s}$ LPS treated group. (ACP-Acid phosphatise). 
(k)

\begin{tabular}{|c|c|c|c|c|c|c|c|c|c|}
\hline \multirow[t]{2}{*}{ Group } & \multirow{2}{*}{$\begin{array}{l}\text { Treatment } \\
\text { LPS(mg/ml) }\end{array}$} & \multirow{2}{*}{$\begin{array}{l}\text { Plant } \\
\text { extract } \\
\text { (mg/ml) }\end{array}$} & \multicolumn{7}{|c|}{ ALP $(\mu \mathrm{mole} p N P$ released $/ \mathrm{min} / \mathrm{mg}$ protein $)$} \\
\hline & & & at $0 \mathrm{~min}$ & $\begin{array}{l}\text { at } 30 \\
\text { min }\end{array}$ & at $1 \mathrm{~h}$ & at $2 \mathrm{~h}$ & at $4 \mathrm{~h}$ & at $8 \mathrm{~h}$ & at $24 \mathrm{~h}$ \\
\hline Control & - & - & $1.14 \pm 0.07$ & $1.14 \pm 0.78$ & $1.13 \pm 0.09$ & $1.13 \pm 0.70$ & $1.13 \pm 0.11$ & $1.12 \pm 0.02$ & $1.12 \pm 0.13$ \\
\hline LPS Treated & 0.05 & - & $1.14 \pm 0.10$ & $1.18 \pm 0.64$ & $1.23 \pm 0.35$ & $1.57 \pm 0.36^{*}$ & $1.59 \pm 0.16$ & $1.65 \pm 0.58^{\mathrm{a}}$ & $1.77 \pm 0.19$ \\
\hline Plant Treated & - & 0.05 & $1.14 \pm 0.73$ & $1.14 \pm 0.25$ & $1.14 \pm 0.36$ & $1.13 \pm 0.86$ & $1.15 \pm 0.17$ & $1.16 \pm 0.10$ & $1.18 \pm 0.12^{*}$ \\
\hline \multirow[t]{5}{*}{ LPS+Plant treated } & 0.05 & 0.01 & $1.13 \pm 0.16$ & $1.17 \pm 0.06$ & $1.22 \pm 0.37$ & $1.56 \pm 0.12$ & $1.54 \pm 0.17$ & $1.62 \pm 0.48$ & $1.72 \pm 0.69$ \\
\hline & & 0.02 & $1.14 \pm 0.19$ & $1.14 \pm 0.47$ & $1.21 \pm 0.08$ & $1.54 \pm 0.06$ & $1.52 \pm 0.17^{*}$ & $1.59 \pm 0.15$ & $1.54 \pm 0.11$ \\
\hline & & 0.03 & $1.14 \pm 0.22$ & $1.12 \pm 0.48$ & $1.19 \pm 0.39$ & $1.39 \pm 0.66$ & $1.47 \pm 0.17$ & $1.41 \pm 0.38^{\mathrm{a}}$ & $1.37 \pm 0.09$ \\
\hline & & 0.04 & $1.15 \pm 0.25$ & $1.13 \pm 0.06$ & $1.14 \pm 0.77$ & $1.24 \pm 0.28^{*}$ & $1.27 \pm 0.19$ & $1.28 \pm 0.10$ & $1.24 \pm 0.71$ \\
\hline & & 0.05 & $1.14 \pm 0.28$ & $1.12 \pm 0.72$ & $1.14 \pm 0.31$ & $1.19 \pm 0.11^{\mathrm{a}}$ & $1.24 \pm 0.02$ & $1.29 \pm 0.33$ & $1.20 \pm 0.74$ \\
\hline
\end{tabular}

Values are represented as mean $\pm \mathrm{SD}(\mathrm{n}=6) .{ }^{*} \mathrm{p}<0.05,{ }^{* *} \mathrm{p}<0.001 \mathrm{v} / \mathrm{s}$ control group; ${ }^{\mathrm{a}} \mathrm{p}<0.05,{ }^{\mathrm{b}} \mathrm{p}<0.001 \mathrm{v} / \mathrm{s}$ LPS treated group. (ALP-Alkaline Phosphatase).

\section{DISCUSSION}

The present investigative study was conducted to determine toxic effects of LPS under in vitro environment by studying various biochemical parameters which indicate oxidative stress, tissue damage or organ failure.

Our results showed that addition of $0.05 \mathrm{mg} / \mathrm{ml}$ LPS showed significant alteration in LPO, protein content, SOD, CAT, GSH, GST and GPx. The alteration in the levels of biochemical parameters may be because of liberation of ROS due to LPS-induced toxicity. LPS intoxication leads to marked increase in lipid peroxidation (table 1 (b)), which is considered as an important action in triggering the manifestation of various serious disorders. Lipid peroxidation is a process which occurs in the presence of some reactive oxygen species and it is a chain-reaction which is self-propagating, the initial oxidation of few lipid molecules can cause tissue damage [22]. The present study also showed the generation of ROS which further causes cellular toxicity. Lipid peroxidation consequently leads to the breakdown of lipids and to the formation of primary oxidation and secondary products including MDA, which can further react with thiol and amino groups, the aldehydes are known to have more diffusing ability than free radicals, which signifies that the damage can be extended to even distant sites. SODs carry out defensive action against reactive oxygen species (ROS)-triggered injury [23]. The experimental findings showed that there is a decrease in SOD levels in the LPS-treated group (table 1(c)). SOD may serve as an inhibitory agent of neutrophil-mediated inflammation and may stand for a novel restorative response for the ROS-dependent tissue injury induced by neutrophils through several mechanisms [24]. Extracellular Superoxide Dimutase, Mn-SOD and $\mathrm{Cu}, \mathrm{Zn}$-SOD have been considered as potent inhibitor of inflammatory cascade by researchers [25-27].

The present experimental study was planned to analyse the activity of catalase against LPS-induced toxicity (table 1 (d)). Catalase is the major antioxidant enzyme which play key role in conversion of $\mathrm{H}_{2} \mathrm{O}_{2}$ into water and $\mathrm{O}_{2}$. Altered catalase activity renders disrupted removal of reactive oxygen species [28]. Over expression of catalase enzyme has been demonstrated to alleviate ROS-induced cell damage while lower levels of catalase promote autophagic cell death $[29,30]$. Glutathione, GST and GPx (Glutathione peroxidase) play remarkable role in protection mechanisms against hepato-toxicity. There was lower expression of GST in LPS-treated group (table 1(f)). Glutathione Peroxidase (GPx) (table 1 (g)) helps in detoxification of peroxides with GSH by acting as an electron donor. During oxidative stress, GPx converts GSH into GSSG whereas another enzyme GR (Glutathione reductase) causes reversible conversion of GSH [31]. Previously published report suggests that the LPS intoxication leads to severe oxidative stress and significant depletion in the levels of GSH shown in table 1 (e) [32]. Since reactive oxygen species mediated oxidative imbalance have been clearly studied that could be responsible for hepatic toxicity induced by LPS, hence, ALT activity (table 1(i)) was much higher than the AST activity (table 1(h)) in hepato-toxic conditions as observed after LPS intoxication in mice. ALP (Alkaline phosphatase) and ACP (Acid phosphatase) (table $1(j)$ ) both have tremendous importance in diagnosis of hepatic deterioration. ALP occurs in sinusoidal and bile canaliculi membranes of liver and is also associated with numerous biological activities (like protein synthesis, metabolic transport across cell membrane, secretary activity and glycogen metabolism) [33, 34]. The alteration in ALP activity (table $1(\mathrm{k})$ ) is linked with the damage in membrane permeability that leads to cell damage.

Medicinal plants are widely used for the treatment of various ailments. Prosopis cineraria is a folk remedy for various diseases for major concern. Our results revealed maximum amelioration at $0.04 \mathrm{mg} / \mathrm{ml}$ concentration of plant extract under in vitro conditions. The study showed that after $2 \mathrm{~h}$ of incubation the levels of biochemical and oxidative stress parameters were altered noticeably in samples analyzed after $2 \mathrm{~h}$ and $4 \mathrm{~h}$ of LPS treatment, in comparison to $0 \mathrm{~min}, 30$ min and $1 \mathrm{~h}$ samples. However, the samples retrieved after $8 \mathrm{~h}$ and 24 $\mathrm{h}$, the levels of the studied parameters showed marginal difference. The ameliorative effect of bark extract was prominent in the samples retrieved after $2 \mathrm{~h}$ incubation. The antioxidant activity of $P$. cineraria is mainly due to the presence of significant amounts of flavonoids, phenolic compounds and thereby, this plant protects from several inflammatory diseases. Flavonoids are known to possess wide array of biological activities, such as anti-oxidative, enzyme inhibiting, apoptosis-inducing, cell-proliferation-inhibiting, and antibacterial [35]. Phytochemical profiling of the crude extracts of the P. cineraria unraveled the presence of phyto-constituents-quercetin and apigenin. Quercetin is proven to mediate anti-inflammatory reactions in paw edema induced by carrageenan in rats [36].

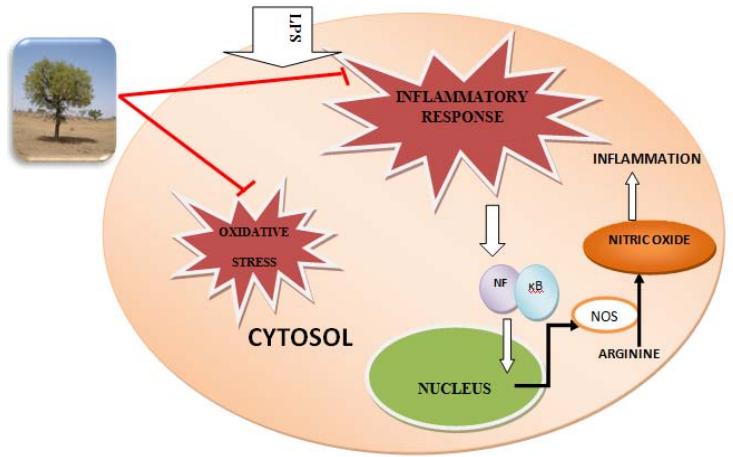

Fig. 1: Diagrammatic Representation of action of Prosopis cineraria against LPS-induced inflammation (imaged by Veena Sharma and Preeti Sharma)

It has been postulated that the curative effects of quercetin are due to its antioxidative and free-radical scavenging potential, thereby restoring the oxidative stress mechanisms under the inflammatory status [37]. Moreover, quercetin has been validated to inhibit both proliferation and activation of macrophage under in vitro conditions by blocking the activation of lipopolysaccharide-induced nuclear factor B (NF-KB) signalling [38]. Similar to flavonoids, apigenin has been shown to exert anti-inflammatory potential by lowering 
oxidative stress though prevention of the expression of several inflammatory factors [39]. It is reported that polyphenols exert antiinflammatory effects. Since, bark extract of $P$. cineraria is rich in polyphenols such as tannins and flavonoids, the anti-inflammatory effect of plant extract may be rendered due to the presence of polyphenolic compounds [40].

\section{CONCLUSION}

In vitro studies are indispensable tool to carry out research for understanding the underlying mechanism with minimal use of animals. The present research work unravelled the alleviating potential of hydro-ethanol extract of Prosopis cineraria against LPSinduced toxicity by combating oxidative stress under in vitro environment. The alteration in the studied parameters were observed at $0.050 \mathrm{mg} / \mathrm{ml}$ LPS concentration; but administration of hydro-ethanol plant extract at concentration $0.04 \mathrm{mg} / \mathrm{ml}$ effectively reduced its level when compared to LPS treated samples under in vitro conditions. Prosopis cineraria is a store house of various phytoconstituents like tannins, steroids, flavone derivatives (namely Prosogerin A, B, C and E), Rutin, Patulitrin, Luteolin, Patuletin, alkaloids etc. which possess anti-oxidative and anti-inflammatory potential and thereby rendering curative role against LPS-induced cell damage. Elaborate in vivo studies can be carried in future in order to validate the in vitro findings and also, to isolate and characterize such potent active principles present in the stem bark extract of Prosopis cineraria which would help in developing herbal formulation for the treatment of inflammation and sepsis.

\section{ACKNOWLEDGMENT}

The authors are extremely grateful and wish to acknowledge the support granted from the Banasthali Vidyapith, Rajasthan for conducting experimental research work

\section{AUTHORS CONTRIBUTIONS}

All the author have contributed equally.

\section{CONFLICT OF INTERESTS}

\section{Declared none}

\section{REFERENCES}

1. Callery MP, Kamei T, Flye MW. Kupffer cell blockade increases mortality during intra-abdominal sepsis despite improving systemic immunity. Arch Surg 1990;125:36-41.

2. Rietschel ET, Holst O, Brade L, Muller Loennies S, Mamat U, Zahinger $\mathrm{U}$, et al. Bacterial endotoxin: chemical constitution, biological recognition, host response and immunological detoxification. Curr Top Microbiol Immunol 1996;216:39-81.

3. Berczi I, Bertok I, Bereznai T. Comparative studies on the toxicity of Escherichia coli lipopolysaccharide endotoxin in various animal species. Can J Microbiol 1966;12:1070-1.

4. Park H, Jung HY, Park EY, Kim J, Lee WJ, Bae YS. Cutting edge: direct interaction of TLR4 with NAD(P)H oxidase 4 isozyme is essential for lipopolysaccharide-induced production of reactive oxygen species and activation of NF-kappa B. J Immunol 2004;173:3589-93.

5. Weiss SJ. Tissue destruction by neutrophils. N Engl J Med 1989;320:365-76.

6. Halliwell B. Free radicals, antioxidants and human disease: curiosity, cause or consequence. Lancet 1994;344:721-4.

7. Prins JM. Antibiotic induced release of endotoxin-clinical data and human studies. J Endotoxin Res 1996;3:269-73.

8. Kalwar SC, Sharma ML, Gurjar RD, Khandelwal MK, Wadhawan SK. Geomorphology and environmental sustainability. Concept Publishing Company, New Delhi, India; 2005.

9. Velmurugan V, Arunachalam G, Ravichandran V. Anthelmintic potential of prosopis cineraria (linn.) druce stem barks. Asian J Plant Sci Res 2011;1:88-91.

10. Kirtikar KR, Basu BD. Indian Medicinal Plants, International Book Distributors: Dehadun, India; 1984. p. 2.

11. Velioglu YS, Mazza G, Gao L, Oomah BD. Antioxidant activity and total phenolics in selected fruits, vegetables and grain products. J Agric Food Chem 1998;46:4113-7.

12. Garg A, Mittal SK. Review on prosopis cineraria: a potential herb of thar desert. Drug Invent 2013;5:60-5.
13. Nwanjo HU, Ojiako OA. Effect of vitamins $E$ and $C$ on exercise induced oxidative stress. Global J Pure Appl Sci 2005;12:199-202.

14. Marklund S, Marklund G. Involvement of superoxide anion radical in the auto-oxidation of pyrogallol and a convenient assay for superoxide dismutase. Eur J Biochem 1974;47:469-74.

15. Aebi H. Catalase in vitro. Methods Enzymol 1984;105:121-6.

16. Ellman GL. Tissue sulfhydryl groups. Arch Biochem Biophys 1959;82:70-7.

17. Habig WH, Pabst MJ, Jakoby WB. Glutathione s-transferases. The first enzymatic step in mercapturic acid formation. J Biol Chem 1974;249:7130-9.

18. Rotruck JT, Pope AL, Ganther HE, Swanson AB, Hafeman DG, Hoekstra WG. Selenium: biochemical role as a component of glutathione peroxidases. Science 1973;179:588-90.

19. Lowry OH, Rosenbrough AL, Farr AL, Randall RJ. Protein measurement with folin phenol reagent. J Biol Chem 1951;193:265-75.

20. Reitman S, Frankel AS. A colorimetric method for the determination of serum glutamic oxaloacetic and glutamic pyruvic transaminase. Am J Clin Pathol 1957;28:53-6.

21. Sadashivam S, Manickam A. Biochemical methods. $2^{\text {nd }}$ edition; 1996;2:121-4.

22. Esterbauer H, Dieber Rotheneder M, Waeg G, Striegl G, Juergens G. Biochemical structural and functional properties of oxidized low-density lipoprotein. Chem Res Toxicol 1990;3:77-92.

23. Kangralkar VA, Patil SD, Bandivadekar RM. Oxidative stress and diabetes: a review. Int J Pharm Appl 2010;1:38-45.

24. Yasui K, Baba A. Therapeutic potential of superoxide dismutase (SOD) for resolution of inflammation. Inflamm Res 2006;55:359-63.

25. Bowler RP, Nicks M, Tran K, Tanner G, Chang LY, Young SK. Extracellular superoxide dismutase attenuates lipopolysaccharide-induced neutrophilic inflammation. Am J Respir Cell Mol Biol 2004;31:432-9.

26. Joseph A, Li Y, Koo HC, Davis JM, Pollack S, Kazzaz JA. Superoxide dismutase attenuates hyperoxia-induced interleukin-8 induction via AP-1. Free Radical Biol Med 2008;45:1143-9.

27. Porfire AS, Leucuța SE, Kiss B, Loghin F, Parvu AE. Investigation into the role of $\mathrm{cu} / \mathrm{Zn}$-SOD delivery system on its antioxidant and anti-inflammatory activity in rat model of peritonitis. Pharmacol Rep 2014;66:670-6.

28. Perez Rivero G, Ruiz Torres MP, Diez Marques ML, Canela A, Lopez Novoa JM, Rodriguez Puyol M. Telomerase deficiency promotes oxidative stress by reducing catalase activity. Free Radical Biol Med 2008;45:1243-51.

29. Ito K, Nakazato T, Yamato K, Miyakawa Y, Yamada T, Hozumi N. Induction of apoptosis in leukemic cells by homovanillic acid derivative, capsaicin, though oxidative stress: implication of phosphorylation of p53 at Ser-15 residue by reactive oxygen species. Cancer Res 2004;64:1071-8.

30. Yu L, Wan F, Dutta S, Welsh S, Liu Z, Freundt E. Autophagic programmed cell death by selective catalase degradation. Proc Natl Acad Sci USA 2006;103:4952-7.

31. Abdalla MY. Glutathione as a potential target for cancer therapy; more or less is good? (mini-review). Jordan J Biol Sci 2011;4:119-24.

32. Nada SA, El-Shamarka ME-S, Omara EA, Abdel-Salam OM. Grape seed extract and vitamin $\mathrm{c}$ combination blocked phytoconstituents-induced multiple organ toxicity in mice. ROS 2019;7:161-75.

33. Hägerstrand I. Distribution of alkaline phosphatase activity in healthy and diseased human liver tissue. Acta Pathol Microbiol Scand A 1975;83:519-26.

34. Jahan MS, Vani G, Shyamaladevi CS. The anti-carcinogenic effect of Solanum trilobatum in diethylnitrosamine induced and phenobarbital promoted hepatocarcinogenesis in rats. Asian J Biochem 2011;6:74-81.

35. Khatri A, Rathore A, Patil UK. Assessment of anti-inflammatory activity of bark of prosopis cineraria (L.) druce. Int J Pharm Res 2012;4:27-9.

36. Rotelli AE, Guardia T, Juarez AO, de la Rocha NE, Pelzer LE. Comparative study of flavonoids in experimental models of inflammation. Pharmacol Res 2003;48:601-6. 
37. Galvez I, de la Cruz JP, Zarzuelo A, Sanchez de Medina FJ, Jimenez J Sanchez, de la Cuesta F. Oral administration of quercitrin modifies intestinal oxidative status in rats. Gen Pharmacol 1994;25:1237-43.

38. Comalada M, Camuesco D, Sierra S, Ballester I, Xaus J, Galvez J, et al. In vivo quercitrin anti-inflammatory effect involves the release of quercetin, which inhibits inflammation through down-regulation of the NF-kappa B pathway. Eur J Immunol 2005;35:584-92.

39. Remick D. Applied molecular biology of sepsis. J Crit Care 2005:10:198-212
40. Sawatzky D, Willoughby D, Colville Nash P, Rossi A. The involvement of the apoptosis-modulating proteins Erk 1/2, Bcl$\mathrm{xL}$, and $\mathrm{Bax}$ in the resolution of acute inflammation in vivo. Am J Pathol 2006;168:33-41.

41. Ulevitch R, Tobias P. Receptor-dependent mechanisms of cell stimulation by bacterial endotoxin. Ann Rev Immonol 2006;13:437-57.

42. Sachdeva S, Kaushik V, Saini V. A review on the phytochemical and pharmacological potential of Prosopis cineraria. Int J Ethnobiol Ethnomed 2014;1:1-4 